\title{
ATMOSPHERIC EFFECTS ON EARTH ROTATION AND POLAR MOTION
}

\author{
David A. Salstein \\ Atmospheric and Environmental Research, Inc. \\ 840 Memorial Drive \\ Cambridge, MA 02139 USA
}

\begin{abstract}
The variability in the earth's rotation rate not due to known solid body tides is dominated on time scales of about four years and less by variations in global atmospheric angular momentum ( $M$ ), as derived from the zonal wind distribution. Among features seen in the length of day $(\Delta 1$. o.d.) record produced by atmospheric forcing are the strong seasonal cycle, quasi-periodic fluctuations around 40-50 days, and an interannual signal forced by a strong Pacific warming event, known as the El Niño. Momentum variations associated with these time scales arise in different latitudinal regions. Furthermore, winds in the stratosphere make a particularly important contribution to seasonal variability.

Other related topics discussed here are (i) comparisons of the $M$ series from wind fields produced at different weather centers, (ii) the torques that dynamically link the atmosphere and earth, and (iii) longer-term non-atmospheric effects that can be seen upon removal of the atmospheric signal. An interesting application for climatological purposes is the use of historical earth rotation series as a proxy for atmospheric wind variability prior to the era of upperair data. Lastly, results pertaining to the role of atmospheric pressure systems in exciting rapid polar motion are presented.
\end{abstract}

\section{INTRODUCTION}

The earth's variable rotation rate and polar motion have been measured accurately in recent years by a number of techniques, including VLBI. Such observations have been accompanied by renewed interest in the geophysical causes of these variations, including those originating within atmospheric winds and pressure systems. Earlier reviews of the subject by Munk and Macdonald (1960) and Lambeck (1980) pointed to the atmosphere for explanations of the observed seasonal variability in earth rotation. With the advent of modern atmospheric data in combination with new geodetic data sets, studies of the earth/atmosphere momentum budget have become possible on a much finer time scale. At

401 
operational agencies such as the U.S. National Meteorological Center (NMC) and the European Centre for Medium Range Weather Forecasts (ECMWF) regularly gridded atmospheric analysis fields are produced on a twicedaily basis as a by-product of their weather forecasting mission; furthermore, these fields have been determined to be sufficiently accurate for geodetic applications.

Recent studies have demonstrated that the expected proportionality between changes in the length of day $(\Delta 1.0 . d$.$) not due to known tides$ and changes in global atmospheric angular momentum (M) exists on time scales of less than a few years (Hide et al., 1980; Rosen and Salstein, 1983). Thus many of the variations found in the earth's rotation are due to phenomena of meteorological origin. In addition, the atmosphere appears to play a role in the excitation of polar motion, due largely to shifts of mass associated with pressure systems.

\section{TEMPORAL VARIABILITY IN M AND $\triangle \mathrm{L} .0 . \mathrm{D}$.}

If the solid earth and the atmosphere form a two-body closed dynamical system, then changes in the momentum about the polar axis of one component must be matched by equal, but opposite changes in the momentum of the other. Based on this simple model, Rosen and Salstein (1983) derived the following linear relationship between $\Delta M$ and $\Delta 1.0 . d$.:

$$
\Delta 1 . \text { o.d. }=1.68 \times 10^{-29} \Delta \mathrm{M}
$$

where $\Delta 1.0 . d$. is in units of seconds and $\Delta M$ is in $\mathrm{kg} \mathrm{m}^{2} \mathrm{~s}^{-1}$. The quantity $M$ is given by the following expression:

$$
M=\frac{2 \pi a^{2}}{g} \iint_{\text {whole atmosphere }}[u] \cos ^{2} \phi d \phi d p
$$

where $a$ is the mean radius of the earth $\left(6.37 \times 10^{6} \mathrm{~m}\right), \mathrm{g}$ is the acceleration due to gravity $\left(9.81 \mathrm{~m} \mathrm{~s}^{-2}\right), \phi$ is latitude, $p$ is pressure and $[u]$ the zonal mean zonal (eastward) wind. In calculations of $M$, the vertical extent of the pressure integration has varied in different data sets. The most complete data set has incorporated levels between the the 1000 and the $1 \mathrm{mb}$ level, or 99.98 of the atmosphere. Since [u] values are not routinely available higher than 100 or $50 \mathrm{mb}$, winds at the high stratospheric levels required special processing.

Figure 1 shows the striking agreement between $M$ and $\Delta 1.0 . d$. series for a four-year period. Evident in both series is the strong seasonal cycle; indeed the angular momentum of the atmosphere varies by nearly a factor of two between its minimum in July/August and its maximum in January/February. The annual signal is largely driven by a summer to winter contrast in tropospheric zonal winds which are greater in the Northern Hemisphere than in the Southern Hemisphere. A semiannual signal is also present, related in part to the presence of that frequency in the tropical stratosphere (Rosen and Salstein, 1985). 
ATMOSPHERIC ANGULAR MOMENTUM VS. LENGTH OF DAY

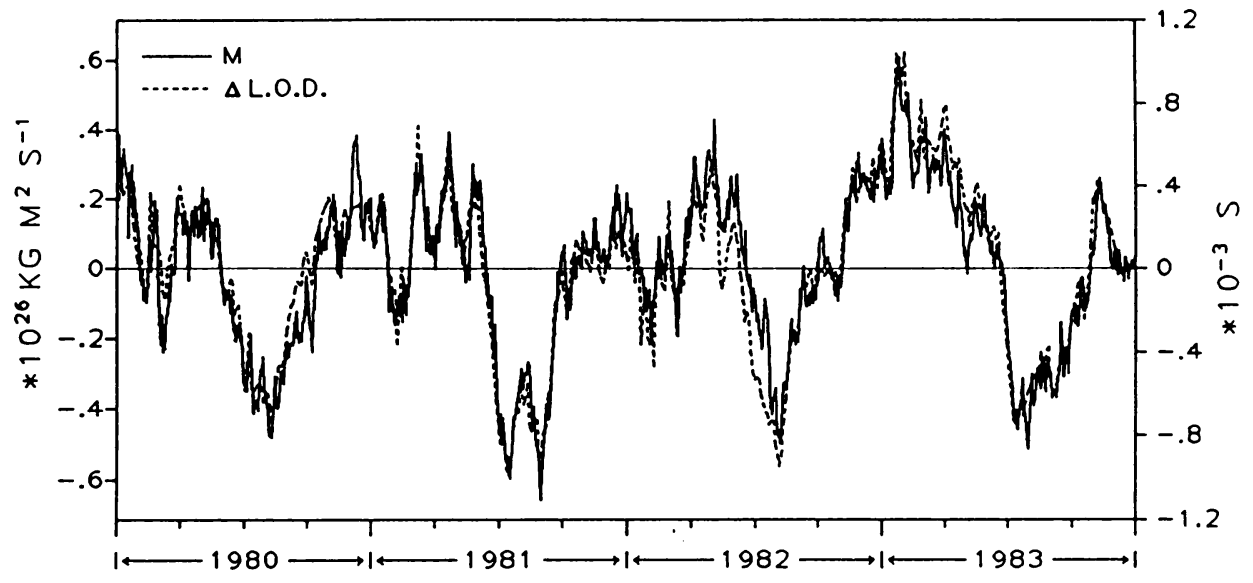

Fig. 1 Time series of daily values of atmospheric angular momentum between 1000 and $1 \mathrm{mb}$ (solid line; scale on left) and three-day means of changes in $\Delta 1.0 . d .$, minus tidal terms. The atmospheric series is based on NMC analyses for 1000-100 mb plus those obtained for 100-1 mb as described by Rosen and Salstein (1985). The $\Delta 1$. o.d. series was produced by Morgan et al. (1985). The mean value of each series has been removed.

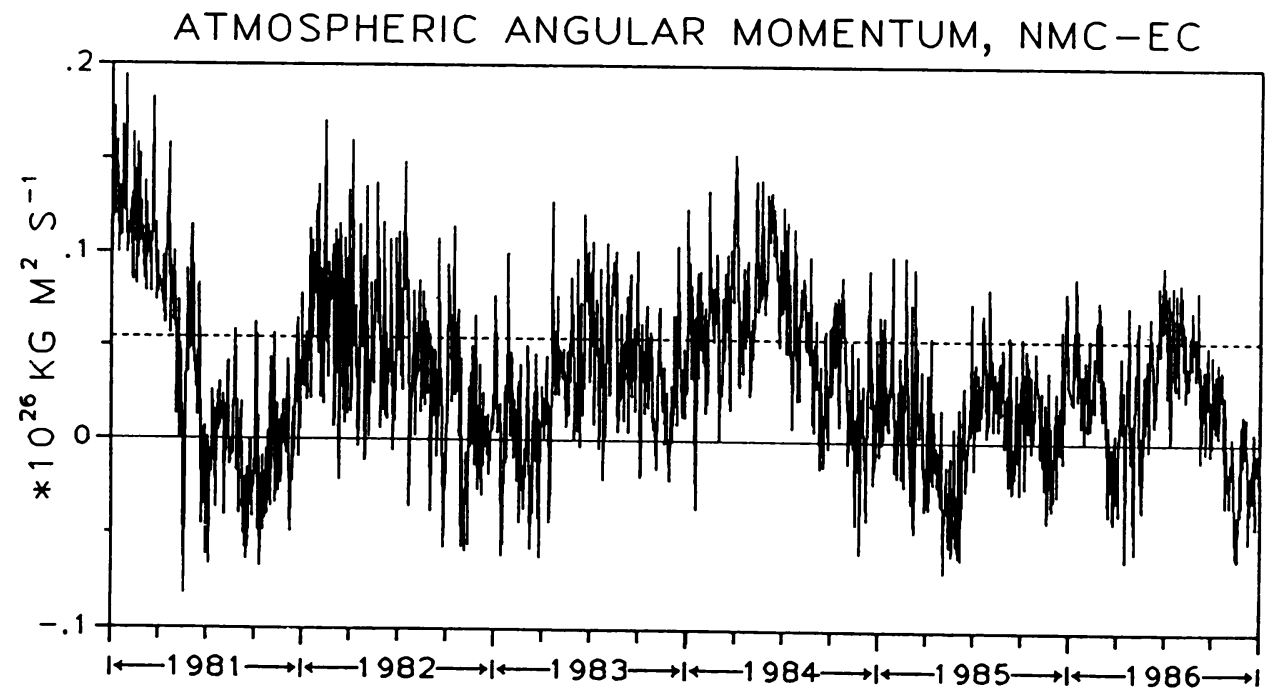

Fig. 2 The difference between time series of daily values of $M$ from the NMC and ECMWF analyses. Values are integrated between 1000 and $50 \mathrm{mb}$. The dashed line indicates the r.m.s. difference between the two series. 
Another pervasive feature in the curves of Fig. 1 arises from the quasi-periodic fluctuations in the atmosphere with periods of around 4050 days (Langley et al., 1981). These fluctuations are the topic of considerable meteorological study and appear to arise in the tropical band, possibly related to convective activity (Madden and Julian, 1972). Furthermore, evidence exists for the propagation of these features into midlatitude regions (Anderson and Rosen, 1983).

A third feature of interest is the interannual variability in $M$ and $\Delta$ l.o.d., and in particular the peak during early 1983 that was related by Rosen et al. (1984) to the strong Pacific Ocean warming event known as the El Niño. Such events are part of one phase of the large-scale Southern Oscillation, a shifting of atmospheric mass across the breadth of the Pacific Ocean with an irregular period of several years. During the 1983 E1 Niño the westerly winds in mid-latitudes were observed to be exceptionally strong and the tropical easterlies weaker than normal; the strong peak in $M$ was the result. A correlation between El Niño/Southern Oscillation (ENSO) events and the earth's rotation rate has been observed both over the past three decades by Chao (1984) and over a century and a quarter using historical data by Salstein and Rosen (1986).

\section{CALCULATION AND COMPARISON OF M SERIES FROM THE WEATHER CENTERS}

The complex system in place at the NMC (Dey and Morone, 1985) is similar to that found at other major weather centers. Meteorological fields are produced on a $2-1 / 2^{\circ} \times 2-1 / 2^{\circ}$ latitude-longitude grid at 12 levels in the vertical by assimilating a large collection of unequally spaced weather observations every 12 hours. This analysis procedure can also incorporate values forecasted by the numerical prediction model and it weights them heavily in data-sparse areas. The analyzed fields are further processed to reduce noise of non-meteorological origin.

Because the systems require a number of complex steps to produce the zonal wind field, used to calculate $M$, an estimate of the error in $M$ can be achieved by comparing the series from a number of weather centers (Rosen and Salstein, 1987). A six-year M difference plot between the NMC and the ECMWF is shown in Fig. 2; the root mean square (r.m.s.) difference between the two series is $5.4 \times 10^{24} \mathrm{~kg} \mathrm{~m}^{2} \mathrm{~s}^{-1}$, equivalent to $0.091 \mathrm{~ms}$ in $\Delta 1.0 . \mathrm{d}$. units. This relatively small error in the analysis of $M$ is comparable to that being achieved in relating $M$ to measurements of $\Delta 1.0 . d$.

\section{DISTRIBUTION OF ANGULAR MOMENTUM VARIABILITY WITHIN THE ATMOSPHERE}

To study regional contributions to the total variability in angular momentum, we have subdivided the atmosphere into a set of 46 equal-area latitudinal belts. In each belt the 10 year series of angular momentum was calculated from zonal winds and separated into three broad frequency bands: interannual, seasonal, and high frequency. The covariance of these series with the global M series, also similarly separated in 
frequency, was calculated, so that at each frequency the sum of the 46 covariance values yields the total variance in $M$. The results are displayed in Fig. 3.

This figure depicts the strong out-of-phase seasonal signal of the subtropical jet streams of the two hemispheres, with a stronger cycle in the Northern Hemisphere. Both the high frequency and interannual signals are relatively evenly contributed by the whole region between $30^{\circ} \mathrm{S}$ and $30^{\circ} \mathrm{N}$.

\section{TORQUES THAT LINK THE EARTH AND ATMOSPHERE}

Angular momentum has been demonstrated to be conserved in the earthatmosphere system by comparing the $M$ and $\Delta 1.0 . d$. series kinematically. However, the physical mechanism, i.e. the torque that dynamically couples the earth and atmosphere is beginning to be studied also, though this problem is less straightforward to formulate. This torque has been computed as the contribution from two separate processes: that from surface friction and the more macroscopic interaction called "mountain torque." The friction torque has been modeled by the use of drag coefficients at the earth's surface while the mountain torque involves knowledge of the atmospheric pressure distribution around topographic features, as well as their elevation.

Recent estimates of the torques remain somewhat inconclusive. On monthly time scales, Wahr and Oort (1984) have found the friction torque to be the more dominant, though they discuss the large uncertainties involved in their computation. On less than monthly time scales, results by Swinbank (1985) and Wolf and Smith (1987) have isolated mountain torques as being the dominant mechanism for rapid transfer of momentum between earth and atmosphere.

\section{HISTORICAL EARTH ROTATION DATA}

\section{AS A PROXY FOR ATMOSPHERIC BEHAVIOR}

The result that changes in $M$ and $\Delta 1.0 . d$. are well correlated on time scales of up to several years enabled Salstein and Rosen (1986) to examine the feasibility of using a historical record of the earth's rotation as a proxy for year-to-year changes in the global zonal wind field. Such an earth rotation series has been prepared by Morrison (1979) and further analyzed by McCarthy and Babcock (1986), and is based mainly on telescopic observations of the occultation of stars by the moon.

The time series of fluctuations in 1.0.d. contains small enough errors to relate to the atmosphere since 1860 , and was filtered to remove the large variations on decadal time scales not of meteorological origin. The resulting filtered time series is shown in Fig. 4; also marked are the years in which a well-defined ENSO warm event occurred, according to a compilation by van Loon and Shea (1985). A student's $t$-test confirms (at the 958 level) that winters in years following ENSO events have "longer days" than otherwise. Since this connection was 


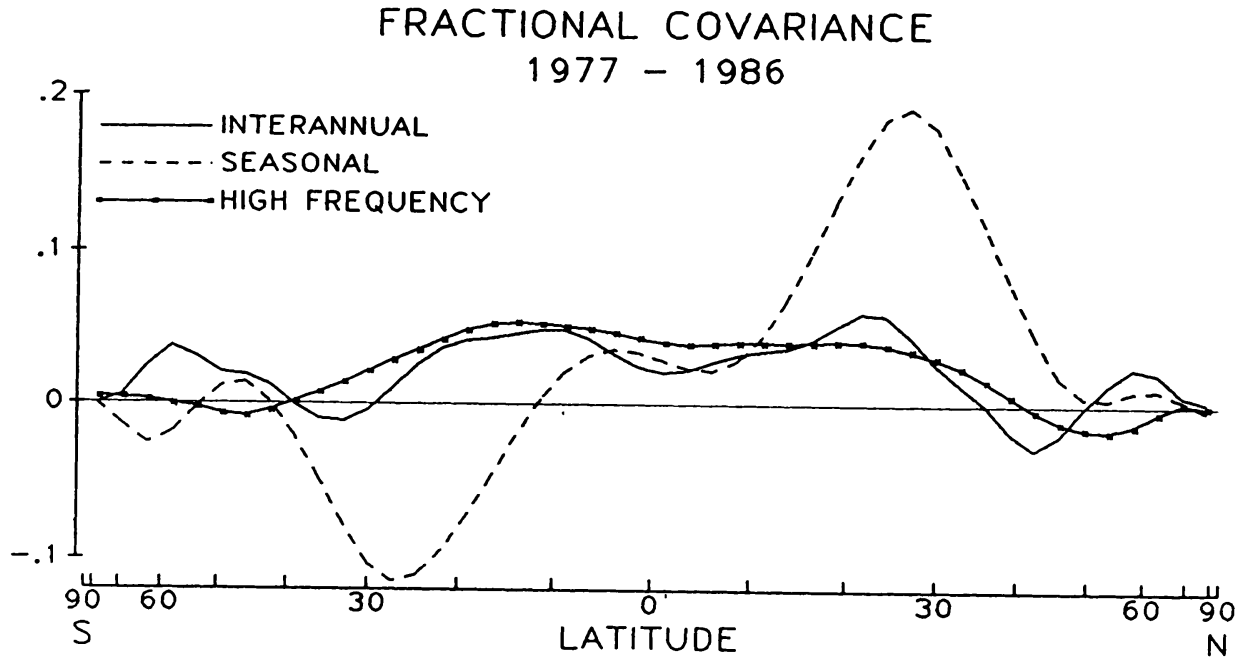

Fig. 3 Fractional part of the variance in the NMC M series for 1977-86 explained by each of 46 equal-area belts for three frequency bands, given by covariance $\left(m_{b}, M\right) \div \operatorname{variance}(M)$, where $m_{b}$ is the belt angular momentum. Each belt's central latitude is at the dots on the high frequency curve.

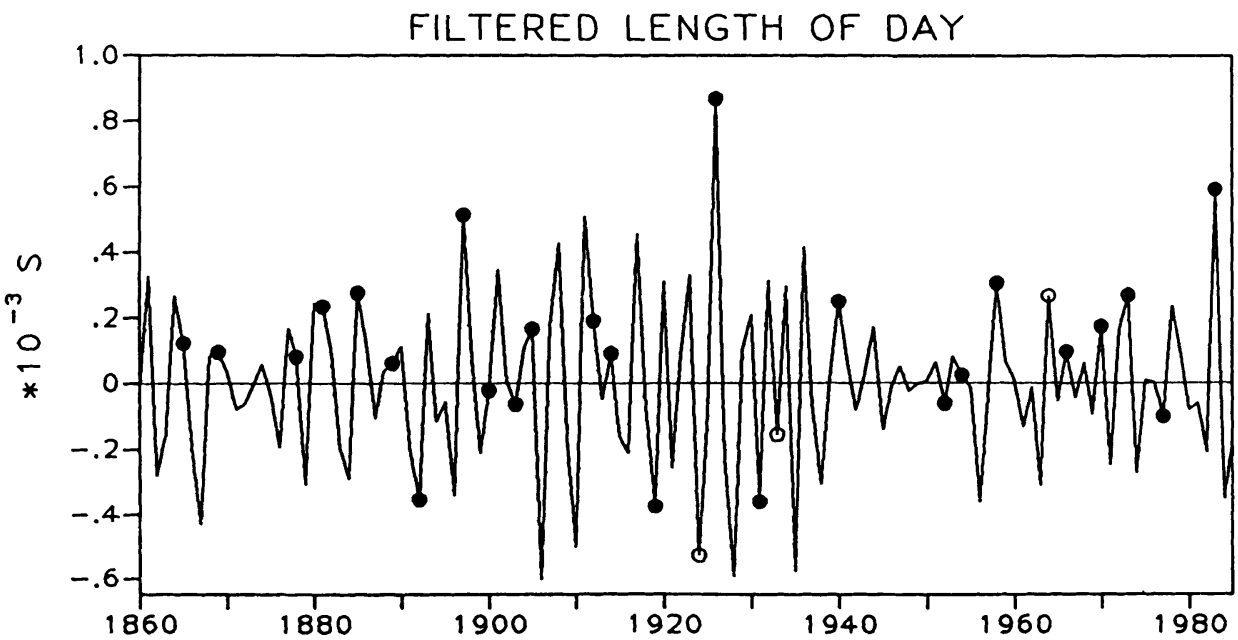

Fig. 4 High-pass filtered values of $\Delta 1.0 . d$. for northern winters during 1860-1985. Solid dots mark those winters that follow a year containing a well-defined ENSO warm event; open dots indicated weak ENSO events. 
expected, we have at least partially justified the use of this historical 1.o.d. series in meteorological studies. We then used the results in Fig. 4 to examine the intra-decadal variability of the implied northern winter global $M$ values. A large contrast was found, for example, between high variability during the 1920 s and low variability of the $1940 \mathrm{~s}$, possibly associated with different temperature regimes during these decades. Based on the interannual curve in Fig. 3, these circulation changes apparently result from zonal wind anomalies in the region between $30^{\circ} \mathrm{S}$ and $30^{\circ} \mathrm{N}$.

\section{ATMOSPHERIC EXCITATION OF POLAR MOTIONS}

As in the model whereby $M$ and $\Delta 1.0 . d$. are closely connected, changes in the equatorial component of atmospheric angular momentum can be related to equatorial changes in the earth rotation vector, that is, the wobble of the polar axis. In this case, however, calculations have indicated that more than the atmosphere needs to be accounted for to produce the observed polar motion.

The largest signals in the polar motion time series are associated with the (approximately) 14-month resonant Chandler wobble and an annual wobble. For quite some time, the annual wobble has been assumed to be largely excited by seasonal fluctuations in the atmosphere's mass (Munk and Macdonald, 1960); however, the forcing mechanism of the Chandler wobble has not been discovered yet, but may also derive some of its power from the atmosphere (Eubanks, personal communication).

Rapid polar motions on the time scale of 15 to 45 days, with smaller amplitudes than the Chandler and seasonal wobbles, have been observed from recent high precision data (Robertson et al., 1985). Using daily NMC analyses and a formulation for computing the atmospheric excitation functions by Barnes et al. (1983), Eubanks et al. (1987) demonstrated that these rapid polar motions are largely driven by surface air pressure changes, as partly modified by the response of sea level to atmospheric loading. Hide (1984) also concluded from the use of ECMWF atmospheric analyses that an overall atmospheric forcing of polar motions was occurring. It should be added here that the Barnes et al. development comprises the effects on polar motion of both the winds and the atmospheric surface pressure distribution modified by the elastic rotational and surface loading deformations of the earth.

We have separated the atmospheric surface pressure excitation values from NMC into a number of equal-area sectors in order to determine which regions are most responsible for behavior on high frequencies of the global excitation. To do so we have calculated the covariance between the regional series and the global series filtered in the 15-45 day high frequency band, and the results are displayed in the map in Fig. 5 (Salstein and Rosen, 1987). As shown in the figure, the main regions contributing to the excitation of rapid polar motions are located over the oceanic storm track regions of the North Pacific and North Atlantic and the midlatitudes of the Southern Hemisphere. 
BAND-PASSED (15-45 DAY) COVARIANCE, $\chi^{P} \quad(1981-1985)$

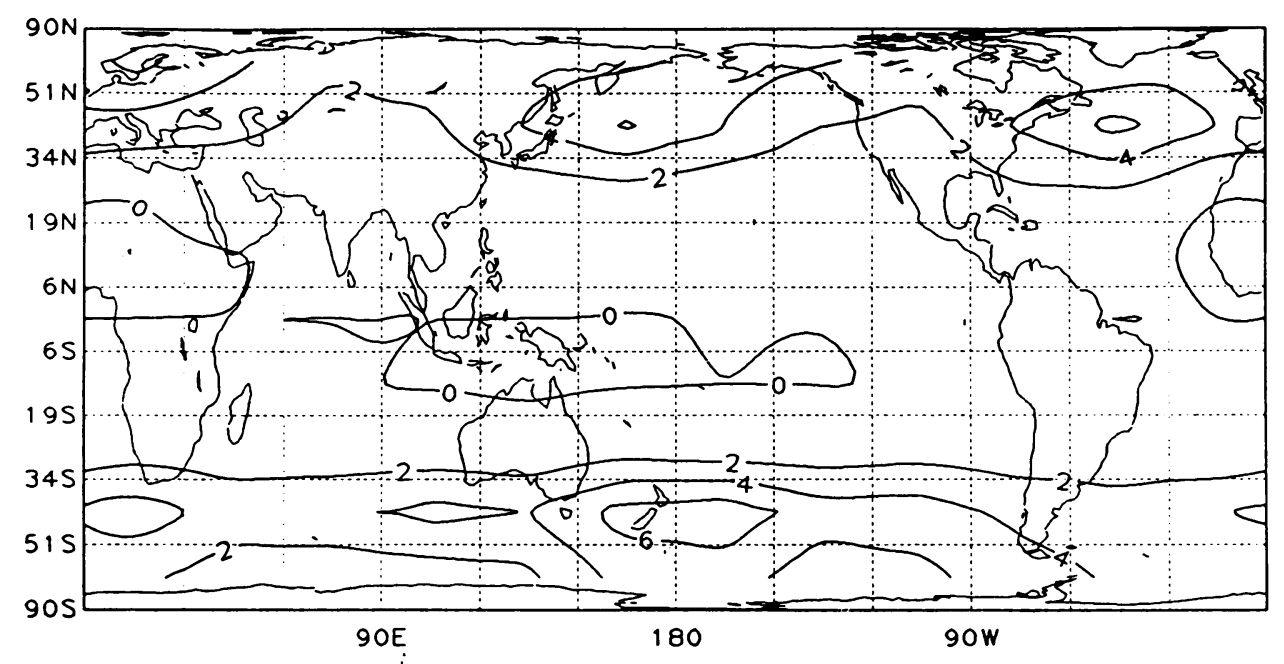

Fig. 5 Covariance between the global atmospheric excitation function for polar motion associated with surface pressure fluctuations $\left(\chi^{\mathrm{P}}\right)$ between -15-45 days and the contribution made to $\chi^{\mathrm{P}}$ in each of 108 equal-area sectors, bounded by the dashed lines. Results are based on NMC analyses for 1981-85, in non-dimensional units of $10^{-16}$.

\section{LENGTH OF DAY - ATMOSPHERIC ANGULAR MOMENTUM}

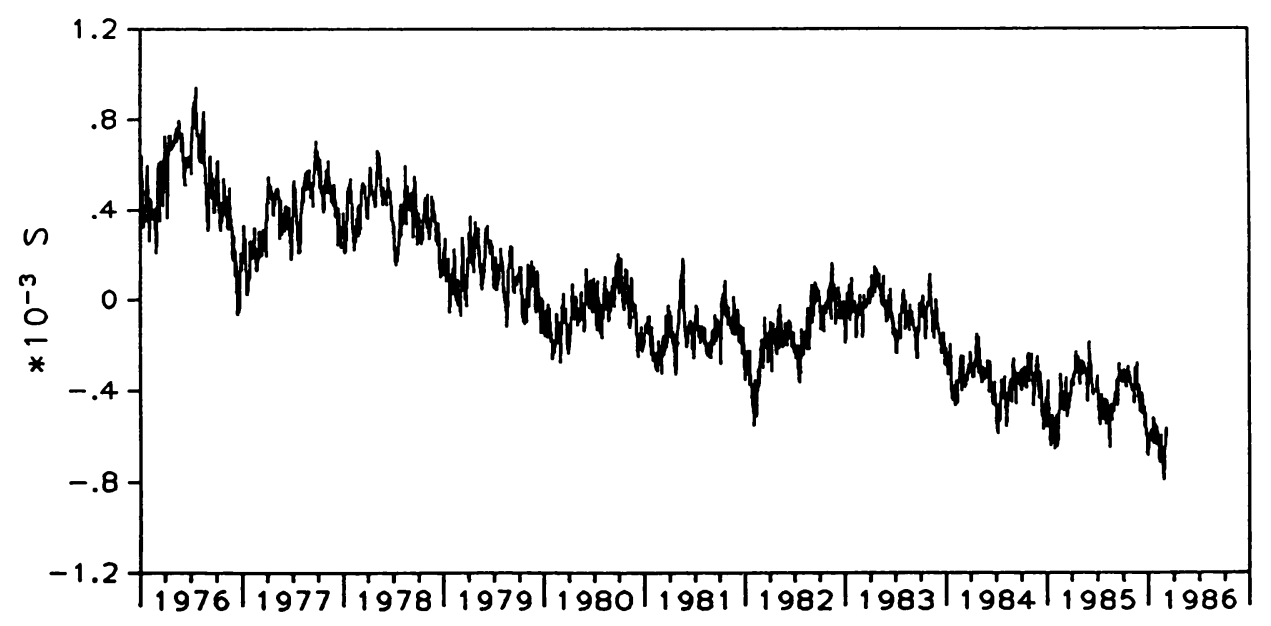

Fig. 6 Difference between 1.o.d. values and $M$ values, in equivalent 1.o.d. units, for 1976 through early 1986, minus a mean. The atmospheric series was based on NMC analyses between 1000 and $100 \mathrm{mb}$, and the 1.o.d. series primarily on one provided by T.M. Eubanks of JPL. 


\section{AFTERWORD}

I have focused here on a number of recent issues and results regarding the role of the atmosphere in both earth rotation and polar motion. The modern atmospheric and geodetic data sets have enabled these studies to progress rapidly.

Knowledge of the atmospheric signal in earth rotation allows a more reliable calculation of the role of non-atmospheric mechanisms, and in particular, the influence of the earth's core. For example, subtraction of the equivalent $M$ series from the $\Delta 1$.o.d. series for the 1976-86 period shows a long period signal which may be modeled by the alternation of relatively rapid decreases with plateau periods (Fig. 6). From such results, a better understanding of the core-mantle interaction may emerge.

A number of other challenges in this field lie ahead including better determination of the Chandler wobble excitation, of the role of the oceans and the hydrological cycle, and improved calculations of earth-atmosphere torques. Advances in both data quality and theoretical formulations will undoubtedly help provide more answers.

\section{ACKNOWLEDGMENTS}

Most of my research reported here has been performed in close collaboration with Dr. Richard D. Rosen of AER Inc., to whom I am much indebted. Support came from the Geodynamics Program of NASA, most recently under contract NAS5-28195.

\section{REFERENCES}

Anderson, J.R. and R.D. Rosen, 1983: 'The latitude-height structure of 40-50 day variations in atmospheric angular momentum.' J.Atmos. Sci., 40, 1584-1591.

Barnes, R.T.H., R. Hide, A.A. White and C.A. Wilson, 1983: 'Atmospheric angular momentum fluctuations, length-of-day changes and polar motion.' Proc. R. Soc. London, A387, 31-73.

Chao, B.F., 1984: 'Interannual length-of-day variation with relation to the Southern Oscillation/E1 Niño.' Geophys. Res. Lett., 11, 541 544

Dey, C.H. and L.L. Morone, 1985: 'Evolution of the National Meteorological Center global data assimilation system: January 1982 - December 1983.' Mon. Wea. Rev., 113, 304-318.

Eubanks, T.M., J.A. Steppe, J.0. Dickey, R.D. Rosen and D.A. Salstein, 1987: 'Rapid polar motions associated with atmospheric pressure changes and oceanic response.' Manuscript in preparation.

Hide, R., N.T. Birch, L.V. Morrison, D.J. Shea, and A.A. White, 1980: 'Atmospheric angular momentum fluctuations and change in the length of the day.' Nature, 286, 114-117.

Hide, R., 1984: 'Rotation of the atmospheres of the Earth and planets.' Phil. Trans. R. Soc. Lond., A313, 107-121. 
Lambeck, K., 1980: The Earth's Variable Rotation. Cambridge University Press, Cambridge, $449 \mathrm{pp}$.

Langley, R.B., R.W. King, I.I. Shapiro, R.D. Rosen and D.A. Salstein, 1981: 'Atmospheric angular momentum and the length of day: a common fluctuation with a period near 50 days.' Nature, 294, 730 732 .

Madden, R.A. and P.R. Julian, 1972: 'Description of global-scale circulation cells in the tropics with a 40-50 day period.' J. Atmos. Sci., 29, 1109-1123.

McCarthy, D.D. and A.K. Babcock, 1986: 'The length of day since 1656.' Phys. Earth and Planet. Inter., 44, 281-292.

Morgan, P.J., R.W. King and I.I. Shapiro, 1985: 'Length of day and atmospheric angular momentum: a comparison for 1981-1983.' J. Geophys. Res., 90, 12645-12652.

Morrison, L.V., 1979: 'Re-determination of the decade fluctuations in the rotation of the earth in the period 1861-1978.' Geophys. J.R. astr. Soc., 58, 349-360.

Munk, W.H. and G.J.F. Macdonald, 1960: The Rotation of the Earth. Cambridge University Press, Cambridge, $323 \mathrm{pp}$.

Robertson, D.S., W.E. Carter, B.D. Tapley, B.E. Schutz, R.J. Eanes, 1985: 'Polar motion measurements: subdecimeter accuracy verified by intercomparison.' Science, 229, 1259-1261.

Rosen, R.D. and D.A. Salstein, 1983: 'Variations in atmospheric angular momentum on global and regional scales and the length of day.' $\mathrm{J}$. Geophys. Res., 88, 5451-5470.

Rosen, R.D. and D.A. Salstein, 1985: 'Contribution of stratospheric winds to annual and semiannual fluctuations in atmospheric angular momentum and the length of day.' J. Geophys. Res., 90, 8033-8041.

Rosen, R.D., D.A. Salstein, A.J. Miller and K. Arpe, 1987: 'Accuracy of atmospheric angular momentum estimates from operational analyses.' Mon. Wea. Rev., 115, in press.

Rosen, R.D., D.A. Salstein, T.M. Eubanks, J.O. Dickey and J.A. Steppe, 1984: 'An E1 Niño signal in atmospheric angular momentum and earth rotation.' Science, $225,411-414$.

Salstein, D.A. and R.D. Rosen, 1986: 'Earth rotation as a proxy for interannual variability in atmospheric circulation, 1860present.' J. Climate Appl. Meteor. , 25, 1870-1877.

Salstein, D.A. and R.D. Rosen, 1987: 'Regional contributions to the atmospheric excitation of rapid polar motions,' manuscript in preparation.

Swinbank, R., 1985: 'The global atmospheric angular momentum balance inferred from analyses made during the FGGE.' Quart. J. R. Met. Soc., 111, 977-992.

van Loon, H. and D.J. Shea, 1985: 'The Southern Oscillation. Part IV: The precursors south of $15^{\circ} \mathrm{S}$ to the extremes of the oscillation.' Mon. Wea Rev., 113, 2063-2074.

Wahr, J.M. and A.H. Oort, 1984: 'Friction- and mountain-torque estimates from global atmospheric data.' J.Atmos. Sci., 41, 190 204.

Wo1f, W.L. and R.B. Smith, 1987: 'Length of day changes and mountain torque during El Niño.' J.Atmos. Sci., in press. 